\title{
Aspectos epidemiológicos, clínicos e olfatórios de pacientes com COVID-19
}

\author{
Epidemiological, clinical and olfactory aspects of patients with COVID-19
}

Aspectos epidemiológicos, clínicos y olfativos de pacientes con COVID-19

Igor Isamu Couceiro Seto ${ }^{1 *}$, Gisele Vieira Hennemann Koury ${ }^{1}$, Vanessa Coutinho Aguiar Gomes ${ }^{1}$, Luigi Ferreira e Silva ${ }^{1}$, Amanda Martins Umbelino ${ }^{1}$, Cíntia Tizue Yamaguchi ${ }^{1}$, Dyana Barbosa Ferreira ${ }^{1}$.

\section{RESUMO}

Objetivo: Avaliar aspectos epidemiológicos, clínicos e olfatórios de pacientes com suspeita/confirmados para COVID-19. Métodos: Estudo transversal, analítico e descritivo, realizado no segundo semestre de 2020, no setor de medicina do trabalho em um complexo hospitalar universitário, em uma cidade do estado do Pará, nos pacientes com suspeita ou confirmação de COVID-19, os quais passaram por uma consulta médica, preenchimento do protocolo de pesquisa e pelo teste olfatório ProFono, no qual se testaram nove aromas diferentes e uma posterior soma dos acertos. Resultados: O gênero feminino foi predominante afetado (79\%); com faixa etária média de 42 anos. A maioria dos pacientes realizou algum exame diagnóstico para COVID $(91 \%)$ e com resultado positivo (91\%). Os sintomas mais prevalentes foram cefaleia, tosse e obstrução nasal. Anosmia foi a alteração olfatória mais incidente (63\%). A azitromicina foi o medicamento mais prescrito para o tratamento $(71 \%)$. Não houve diferença estatística entre os pacientes com anosmia, hiposmia ou sem alteração olfativa na pontuação total do teste olfatório, tampouco mudanças na história natural da doença com os tratamentos propostos. Conclusão: A COVID-19 apresenta sintomas diversificados e grande prevalência de alterações olfatórias que,devido seu grande impacto no cotidiano, deve ser investigada nos portadores desta doença.

Palavras-chave: Anosmia, COVID-19, Pandemia.

\begin{abstract}
Objective: To evaluate epidemiological, clinical and olfactory aspects of patients suspected / confirmed for COVID-19. Methods: Cross-sectional, analytical and descriptive study, carried out in the second half of 2020, in the sector of occupational medicine in a University Hospital Complex, in a city of state of Pará, in patients with suspected or confirmed COVID-19, who underwent a medical consultation, completed the research protocol and the ProFono olfactory test, which tested nine different aromas and a subsequent sum of correct answers. Results: The female gender was predominantly affected (79\%); with an average age group of 42 years. Most patients underwent some diagnostic exam for COVID (91\%) and with a positive result (91\%). The most prevalent symptoms were headache, cough and nasal obstruction. Anosmia was the most frequent olfactory alteration (63\%). Azithromycin was the most prescribed medication for treatment $(71 \%)$. There was no statistical difference between patients with anosmia, hyposmia or without olfactory changes in the total olfactory test score, nor changes in the natural history of the disease with the proposed treatments. Conclusion: COVID-19 has diversified symptoms and a high prevalence of olfactory changes that, due to its great impact on daily life, should be investigated in patients with this disease.
\end{abstract}

Keywords: Anosmya, COVID-19, Pandemic.

${ }^{1}$ Hospital Bettina Ferro de Souza (HUBFS), Belém - PA. *E-mail: igorcouceiro@hotmail.com 


\section{RESUMEN}

Objetivo: Evaluar aspectos epidemiológicos, clínicos y olfativos de pacientes sospechosos / confirmados de COVID-19. Métodos: Estudio transversal, analítico y descriptivo, realizado en el segundo semestre de 2020, en el sector de medicina del trabajo en un complejo hospitalario universitario, en una cidad de Pará, en pacientes con COVID-19 sospechado o confirmado., que se sometió a una consulta médica, completó el protocolo de investigación y la prueba olfativa ProFono, que probó nueve aromas diferentes y una posterior suma de aciertos. Resultados: El sexo femenino se vio afectado predominantemente (79\%); con un grupo de edad promedio de 42 años. La mayoría de los pacientes se sometieron a algún examen diagnóstico para COVID (91\%) y con resultado positivo (91\%). Los síntomas más prevalentes fueron cefalea, tos y obstrucción nasal. La anosmia fue la alteración olfativa más frecuente (63\%). La azitromicina fue el medicamento más recetado para el tratamiento $(71 \%)$. No hubo diferencia estadística entre pacientes con anosmia, hiposmia o sin cambios olfatorios en la puntuación total de la prueba olfativa, ni cambios en la historia natural de la enfermedad con los tratamientos propuestos. Conclusión: COVID-19 presenta síntomas diversificados y una alta prevalencia de cambios olfativos que, por su gran impacto en la vida diaria, deben ser investigados en pacientes con esta enfermedad.

Palabras clave: Anosmia, COVID-19, Pandemia.

\section{INTRODUÇÃO}

Em janeiro de 2020, a Organização Mundial da Saúde (OMS) declarou oficialmente a pandemia da COVID19 (Corona Vírus Disease-19) como uma emergência em saúde pública de interesse global (CAO QD, et al., 2020) e com elevado impacto socioeconômico e cultural.

Atualmente, é aceito que a maioria dos casos da COVID-19 resulta da transmissão inter-humana, por meio de gotículas ou contato direto, com um período médio de incubação de 3 dias (WANG D, et al., 2020), podendo surgir de 2 a 14 dias após exposição (TORRETTA S, et. al., 2020) sintomas que incluem febre, tosse, fadiga, dispneia, mialgia, cefaleia, alterações gastrointestinais, dentre outros (CAO QD, et al., 2020; CHEN N, et al., 2020; MUSTAFA MWM, 2020; WANG D, et al., 2020). Em alguns casos, características mais leves, comumente descritas após infecções do tipo influenza, como hiposmia/anosmia e hipogeusia/ageusia (geralmente secundária a alterações olfativas) também estão presentes (TORRETTA S, et al., 2020) e podem ter apresentação precoce (LOVATO A, et al., 2020).

Nesse contexto pandêmico, as disfunções olfatórias foram reconhecidas como sintomas iniciais e altamente incidentes, com prevalência estimada em cerca de 56\% (PADERNO A, et al., 2020), chegando a índices de 86\% (MENNI C, et al., 2020; MOEIN ST, et al., 2020 e YAN CH, et al., 2020). A importância fisiológica da olfação, no cotidiano, na identificação de fatores ambientais e ameaças potenciais é tão relevante que está relacionada à redução da expectativa de vida, mesmo em indivíduos sem diagnóstico de doença neurodegenerativa (JOFFILY L, et al., 2020). Esta alteração também se mostra importante ao impactar diretamente na qualidade de vida de pacientes que experimentam ausência sensitiva de forma prolongada (temporária ou não).

Os mecanismos implicados na disfunção olfatória frequente em COVID-19 não são totalmente compreendidos, mas as células do epitélio nasal possuem abundantes receptores da enzima conversora de angiotensina 2 (ECA-2), que medeiam a entrada de SARS-CoV-2 (Severy Acute Respiratory Syndrome-2) nas células. A disfunção do neuroepitélio secundária à resposta inflamatória localizada da fenda olfatória pode prejudicar o olfato temporária ou permanentemente (HUMMEL T, et al., 2020), gerando uma alteração de caráter condutivo (YAN CH, et al., 2020).

Finalmente, a causa mais óbvia de anosmia parece ser a de dano direto dos receptores de neurônios olfatórios, já que outros coronavírus humanos foram previamente comprovados por se ligarem diretamente a estes receptores (BILINSKA K, BUTOWT R, 2020). No entanto, alguns autores acreditam que a anosmia se deve, de alguma forma, à inflamação dos nervos olfatórios, e não a danos à estrutura de seus receptores (VILLALBA NL, et al., 2020). 
Seguindo esta busca pelo padrão de neutrotropismo, modelos animais indicam que os coronavírus podem seguir para o cérebro através do nervo olfatório, do bulbo ou ambos, causando dano neuronal ou morte (NETLAND J, et al., 2008), por meio da expressão de TMPRSS2 (Serina Protease Transmembranar 2) e ECA-2 nas células de sustentação neuronais (MOEIN ST, et al., 2020; XYDAKIS MS, et al., 2020).

A emergência de saúde causada pela pandemia exige um máximo empenho dos profissionais envolvidos neste atendimento e necessita de uma reorganização do sistema de saúde para suprir às necessidades excepcionais destes pacientes de maneira eficaz. Isso se traduz em busca incessante do conhecimento fisiopatológico, clínico e terapêutico desta moléstia (ANSARIN M, 2020).

Embora várias infecções virais possam levar às alterações sensoriais olfatórias, não se sabe exatamente como elas se comportam na COVID-19. Diante da prevalência em escala mundial e pelo impacto social causado pelas alterações olfatórias, é muito importante avaliar tais alterações, bem como os aspectos clínicos, epidemiológicos e terapêuticos, a fim de auxiliar na melhor caracterização e tratamento desta nova doença.

\section{MÉTODOS}

Trata-se de um estudo transversal, observacional e de caráter descritivo/analítico. A pesquisa ocorreu em duas instituições públicas assistenciais aos pacientes confirmados ou suspeitos para COVID-19, realizado no segundo semestre de 2020. A população de estudo envolveu funcionários dos serviços participantes que se dirigiram, como pacientes, ao serviço de medicina do trabalho para consulta médica devido à suspeita/confirmação de COVID-19.

Foram inclusos na pesquisa funcionários de todas as idades, gêneros, localidades, ocupações, em qualquer período de evolução da doença, desde que tivessem clínica compatível com COVID-19 de grau leve, confirmados por exames laboratoriais ou não, sem critérios de gravidade estipulados pelo Ministério da Saúde (2020), tais como: dispneia; cianose central; uso de musculatura acessória; saturação de oxigênio inferior a 95\% em ar ambiente; taquipneia > 30 incursões por minuto; hipotensão arterial; alteração no estado mental; piora das condições de doenças de base e persistência de febre (BRASIL, 2020).

Foram excluídos do projeto pacientes que não tiveram clínica compatível, não aceitaram participar mediante apresentação do Termo de Consentimento Livre e Esclarecido (TCLE), apresentavam doenças e/ou alterações olfatórias e gustativas prévias (informação obtida por autorrelato) e estiveram em curso agudizado de condições otorrinolaringológicas (sem relação com COVID).

A pesquisa foi realizada após a aprovação do projeto de pesquisa pelo Comitê de Ética em Pesquisa (CEP) do Complexo Hospitalar da UFPA-EBSERH (Universidade Federal do Pará-Empresa Brasileira de Serviços Hospitalares) (CAAE: 35907020.0.0000.0017), pela instituição proponente e pelos pacientes participantes através da assinatura do TCLE.

Os dados foram obtidos por meio de protocolo de pesquisa preenchidos pelos pacientes nas salas de espera das consultas ambulatoriais. Este protocolo apresentou perguntas referentes às alterações otorrinolaringológicas, principalmente as que comprometiam olfato, bem como parâmetros epidemiológicos e terapêuticos. Após o preenchimento do mesmo, os pacientes foram atendidos em consulta médica para realizar anamnese dirigida para a COVID e definição de conduta clínica.

A olfação foi avaliada através do Instrumento de Avaliação e Treinamento Olfativos Pró-Fono no dia da consulta médica, no qual foram oferecidos 9 aromas para testagem dentre os 15 frascos difusores de aroma com tampas rosqueáveis possíveis (abacaxi, alho, banana, café, canela, cebola, cereja, chocolate, coco, cravo, laranja, maçã, menta, morango e rosa). Após o teste individual de cada frasco (tempo médio estipulado de até 15 segundos por aroma, podendo ser repetido uma única vez, se solicitado pelo paciente), o paciente foi apresentado a um tabuleiro de avaliação olfativa (para reconhecimento e identificação de cada aroma), contendo as ilustrações dos 15 aromas. Feito a discriminação, uma a uma, foi calculado a pontuação final de acertos e erros. 
Todo o material e equipamento utilizados na pesquisa foram manipulados cuidadosamente pelos pesquisadores, seguindo as orientações sanitárias de higiene e precaução vigentes no momento da pesquisa (higienização dos equipamentos com álcool 70\%, paramentação médica completa e uso de máscara obrigatória pelos pacientes, sendo esta retirada no exame físico e no momento da testagem).

A análise estatística foi realizada através do programa Biostat 5.0, no qual foi utilizado teste exato de Fischer e Qui-quadrado para comparação de variáveis categóricas; teste T de Student para comparação de variáveis quantitativas de distribuição normal e teste de Mann-Whitney para comparação de variáveis quantitativa de distribuição não normal.

\section{RESULTADOS}

Participaram da pesquisa 76 pacientes, sendo 66 (87\%) funcionários do HUJBB e 10 (13\%) do HUBFS. Realizaram teste para o diagnóstico da infecção por SARS-COV-2 (teste molecular, sorologia ou teste rápido) 91\% (69/76) dos pacientes, com resultado positivo em 91\% destes (63/69). O tempo decorrido entre o início de sintomas e realização do teste, foi de, em média, 13 dias. Apenas 2 pacientes necessitaram de internação hospitalar (3\%) (Tabela 1).

Tabela 1 - Informações epidemiológicas dos pacientes com suspeita/confirmação de COVID-19, em Belém-PA, 2020.

\begin{tabular}{|c|c|}
\hline Característica & Observação \\
\hline Idade (média \pm DP anos) & $42 \pm 13$ \\
\hline Sexo (M\%/F\%) & $21 \% / 79 \%$ \\
\hline Prevalência de Internação Hospitalar (\%) & $3 \%(2 / 76)$ \\
\hline Realização de Teste para COVID-19 (\%) & $91 \%(69 / 76)$ \\
\hline Positividade do Teste para COVID-19 (\%) & $91 \%(63 / 69)$ \\
\hline Tempo de início da doença ao realizar teste (média \pm DP dias) & $13 \pm 6$ \\
\hline Prevalência de Rinite e/ou Sinusite & $36 \%(27 / 76)$ \\
\hline Prevalência de HAS & $18 \%(14 / 76)$ \\
\hline Prevalência de DM & $6 \%(5 / 76)$ \\
\hline
\end{tabular}

Legenda:DP = Desvio Padrão; $\mathrm{M} \%$ = Porcentagem de Homens; F\% = Porcentagem de Mulheres; HAS = Hipertensão Arterial Sistêmica; DM = Diabetes Mellitus.

Fonte: Seto IIC, et al., 2020.

Entre os sintomas gerais referidos pelos pacientes, a cefaleia foi o predominante, seguida por tosse. Já com relação aos sintomas nasais, o mais prevalente foi o de obstrução nasal (Tabela 2).

Tabela 2 - Sintomas relatados por pacientes com suspeita/confirmação de COVID-19, em Belém-PA, 2020.

\begin{tabular}{lc}
\hline Sintomas & Prevalência \\
\hline Sintomas Gerais & $\%$ \\
\hline Cefaleia & $79 \%(60 / 76)$ \\
Tosse & $64 \%(49 / 76)$ \\
Dor no Peito & $57 \%(43 / 76)$ \\
Odinofagia & $53 \%(40 / 76)$ \\
Tontura & $49 \%(37 / 76)$ \\
Vômito & $36 \%(27 / 76)$ \\
\hline Sintomas Nasais & $\%$ \\
\hline Obstrução nasal & $62 \%(47 / 76)$ \\
Irritação nasal & $54 \%(41 / 76)$ \\
Secreção nasal & $45 \%(34 / 76)$ \\
Ardor nasal & $36 \%(27 / 76)$ \\
\hline
\end{tabular}

Fonte: Seto IIC, et al., 2020. 
Entre os múltiplos esquemas terapêuticos propostos (tratamento medicamentoso foi prescrito para 49 pacientes), a azitromicina foi o mais prescrito (Tabela 3 ).

Tabela 3 - Prevalência dos medicamentos utilizados pelos pacientes com suspeita/confirmação de COVID-19, em Belém-PA, 2020.

\begin{tabular}{ll}
\hline Medicamento & Prevalência \\
\hline Azitromicina & $71 \%(35 / 49)$ \\
Corticoide oral e/ou tópico & $41 \%(20 / 49)$ \\
Hidroxicloroquina & $29 \%(14 / 49)$ \\
Analgésico e/ou AINE & $20 \%(10 / 49)$ \\
Ivermectina & $18 \%(9 / 49)$ \\
\hline
\end{tabular}

Legenda: AINE = Anti-inflamatório não esteroidal.

Fonte: Seto IIC, et al., 2020.

Relataram alterações olfatórias 84\% (64/76) dos pacientes avaliados. A maioria (63\%) apresentou anosmia. As alterações olfativas foram referidas como primeiro sintoma em $39 \%$ dos pacientes com a queixa (18/64) (Figura 1).

Figura 1 - Alterações de olfato em pacientes com suspeita/confirmação de COVID-19, em Belém-PA, 2020.

- HIPOSMIA $\square$ ANOSMIA $\square$ NORMAL

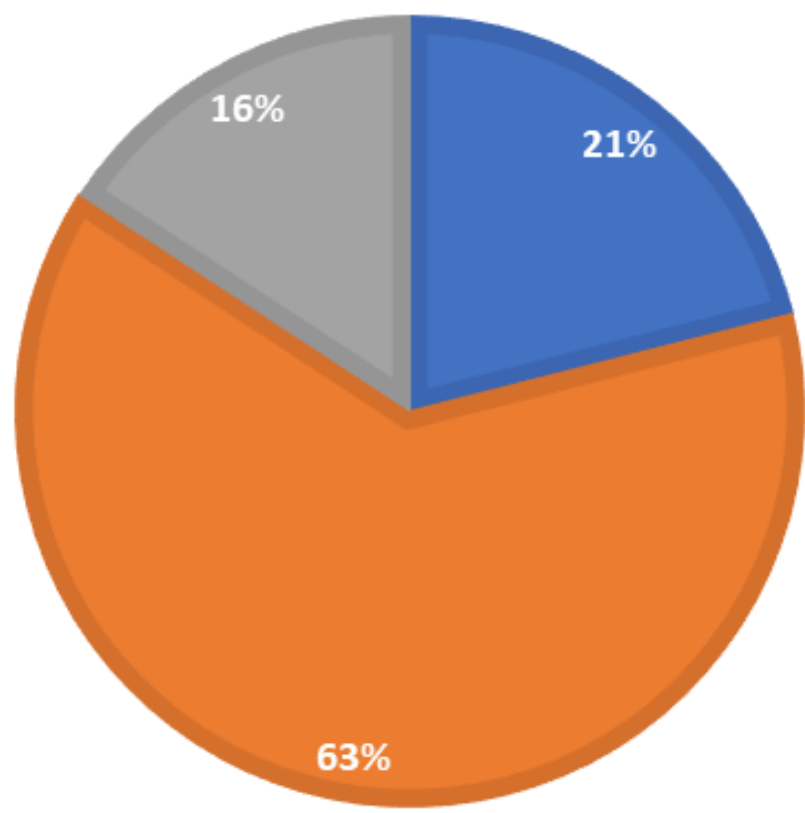

Fonte: Seto IIC, et al., 2020.

Não houve diferença significativamente estatística na pontuação do teste olfativo entre os pacientes com histórico de anosmia, hiposmia ou sem alteração olfativa (Tabela 4).

Tabela 4 - Pontuação no teste olfatório correlacionada com presença de alterações olfatórias em pacientes com suspeita/confirmação de COVID-19, em Belém-PA, 2020.

\begin{tabular}{ccccc}
\hline & $\begin{array}{c}\text { Pacientes com } \\
\text { anosmia } \\
(\mathbf{N}=\mathbf{4 8})\end{array}$ & $\begin{array}{c}\text { Pacientes com } \\
\text { hiposmia } \\
(\mathbf{N}=\mathbf{1 7})\end{array}$ & $\begin{array}{c}\text { Pacientes sem } \\
\text { alteração } \\
\mathbf{( N = 1 1 )}\end{array}$ & $\mathbf{p}$ \\
\hline $\begin{array}{c}\text { Pontuação no Teste } \\
\text { Olfativo }\end{array}$ & $6,0 \pm 1,8$ & $6,1 \pm 2,0$ & $6,2 \pm 1,4$ & 0,925 \\
\hline
\end{tabular}

Fonte: Seto IIC, et al., 2020. 
Não houve diferença, com relevância estatística, na pontuação do teste olfatório entre os pacientes que realizaram algum tipo de tratamento e os demais que não realizaram (Tabela 5).

Tabela 5 - Pontuação no teste olfatório correlacionada com tratamento realizado pelos pacientes com suspeita/confirmação de COVID-19, em Belém-PA, 2020.

\begin{tabular}{lccc}
\hline & $\begin{array}{c}\text { Pacientes que realizaram } \\
\text { tratamento } \\
(\mathbf{N}=\mathbf{4 9})\end{array}$ & $\begin{array}{c}\text { Pacientes que não } \\
\text { realizaram tratamento } \\
\mathbf{( N = 2 7 )}\end{array}$ & $\mathbf{p}$ \\
\hline Pontuação no Teste Olfativo & $6,2 \pm 1,4$ & $5,9 \pm 2,2$ & 0,698 \\
\hline
\end{tabular}

Fonte: Seto IIC, et al., 2020.

\section{DISCUSSÃO}

Pelo contexto pandêmico global, onde todos os setores da sociedade foram intensamente afetados, muitos estudos foram encorajados a fim decompreender melhor os aspectos relevantes dessa moléstia. A começar pelos aspectos epidemiológicos, muitas pesquisas evidenciaram perfis variados entre regiões, mostrando a complexidade da doença.

O presente estudo evidenciou maior acometimento do gênero feminino (79\%), concordando com a prevalência em mulheres encontrados por Lima MA, et al. (2020), onde houve predominância no gênero feminino em $54,4 \%$. Em dados de uma revisão sistemática europeia, também houve maior incidência em mulheres (63\%) (LECHIEN JR, et al., 2020). Duarte MMS, et al. (2020), verificou que as mulheres foram afetadas em $59,8 \%$.

Possivelmente, o maior acometimento do gênero feminino seja reflexo do cuidado maior que as mulheres tendem a ter com sua própria saúde (os pacientes do estudo precisaram procurar o serviço de atendimento especializado) e por serem a maioria em muitas das profissões inclusas neste trabalho (técnicas de enfermagem, recepcionistas e auxiliares administrativas).

Contrariando a maior parte dos estudos, em uma revisão sistemática proposta por Lovato A, et al. (2020), com 1556 pacientes, 895 eram homens (57,5\%) e 661 mulheres (42,5\%). Provavelmente essa prevalência maior em homens seja pelo fato de, nessa revisão sistemática, não haver a necessidade de procura ativa dos mesmos para atendimentos médicos e sim uma estimativa baseada em dados e números estatísticos divulgados por estudos e órgãos competentes.

Com relação a faixa etária dos pacientes envolvidos, observou-se que a idade média foi de 42 anos (desvio padrão de \pm 13 anos), concordando com a maioria dos estudos que evidenciam maior acometimento das quartas e quintas décadas. A média de idade em anos, por estudo, foi de 36 (LECHIEN JR, et al., 2020), 41 (LIMA MA, et al., 2020), 44 (DUARTE MMS, et al., 2020), 47 (GUAN WJ, et al., 2020), 55 (CHEN N, et al., 2020), 56 (WANG D, et al., 2020) e 57 (ZHANG JJ, et al., 2020).

Na população sob estudo, notou-se que a maioria (91\%) realizou testes diagnósticos para COVID-19 (o teste realizado por cada paciente não foi especificado neste estudo) e dentre estes, $91 \%$ obteve resultados positivos. Este estudo segue a tendência de outros realizados, a exemplo do proposto por Kosugi EM, et al. (2020), no qual 72,3\% foram submetidos a testes. Com relação aos índices de positividade, este resultado foi compatível com os achados de Joffily L, et al. (2020), com positividade em $88,8 \%$ dos pacientes e com os resultados de Kosugi EM, et al. (2020), com resultados positivos em 79,2\%.

O tempo médio entre início das queixas clínicas e realização dos exames diagnósticos foi de 13 dias. Entre os pacientes que realizaram teste para diagnóstico, aqueles com resultado positivo apresentavam tempo de sintomas significativamente menor em comparação àqueles com resultado negativo (12 \pm 5 dias $X 22 \pm 10$ dias, $p<0,05)$, possivelmente por terem sintomas mais exuberantes e verificarem necessidade de exames esclarecedores de forma mais precoce. Conforme Lechien JR, et al. (2020), o tempo médio entre o início da infecção e a avaliação foi de $9,2 \pm 6,2$ dias, onde $34,5 \%$ dos pacientes estavam na fase aguda da infecção, enquanto o restante dos pacientes já não apresentavam sintomas gerais. Já Lima MA, et al. (2020), verificou que o tempo médio desde o início dos sintomas até a avaliação médica foi de 4 dias (1-19 dias). 
Com relação às comorbidades mais prevalentes, notou-se o maior número de relatos de rinite/sinusite (36\%), seguida por hipertensão arterial (18\%) e diabetes mellitus (6\%). Em outros estudos, observou-se que as doenças crônicas frequentemente associadas foram hipertensão arterial $(17,4 \%)$, diabetes mellitus $(3,8 \%)$ e doença coronariana (3,8\%) (LOVATO A, et al., 2020). Já as comorbidades mais prevalentes, em um estudo europeu, foram rinite alérgica, asma, hipertensão e hipotireoidismo (LECHIEN JR, et al., 2020).

O perfil deste estudo está de acordo ao observado por Kosugi EM, et al. (2020) ao relatar que 36,4\% dos pacientes apresentavam comorbidades, sendo a rinite a mais frequente $(20,9 \%)$, seguida por asma e hipertensão arterial sistêmica (HAS) com 13 pacientes cada $(5,1 \%)$. Rinossinusite crônica foi relatada por sete pacientes $(2,8 \%)$.

Os sistemas de saúde sobrecarregados apontam a necessidade urgente de desenvolver estratégias para prever os primeiros sintomas e a trajetória da doença com biomarcadores e aspectos clínicos prognósticos (XIE J, et al., 2020). Um método econômico de estratificação precoce do risco da gravidade da doença permitiria uma melhor tomada de decisão médica, rápida e apropriada intervenção para a gravidade, além de facilitar a alocação de recursos médicos limitados (YAN CH, et al., 2020). Daí a necessidade de se qualificar e especificar o comportamento clínico apresentando pela infecção sob estudo, embora seja conhecida a grande variabilidade deste aspecto.

Com relação aos sintomas gerais referidos pelos pacientes, cefaleia foi a queixa mais frequente (79\%), seguida por tosse (64\%), dor no peito (57\%) e odinofagia (53\%), concordantes ao estudo de Lechien JR et al. (2020), no qual sintomas tais quais tosse, mialgia, perda de apetite, diarreia, febre, cefaleia e astenia foram os sintomas mais prevalentes, correspondendo a mais de $45 \%$ dos pacientes. $\mathrm{O}$ estudo brasileiro proposto por Duarte MMS, et al. (2020) apontou febre (83,6\%), tosse $(82,5 \%)$ e dispneia $(74,3 \%)$ como os sinais e sintomas mais frequentes, seguidos de desconforto respiratório $(65,6 \%)$ e dor de garganta (26,8\%). No atual estudo, não concordante com a maior parte dos estudos publicados, febre não foi considerada uma queixa significativa, provavelmente por esquecimento por parte dos entrevistados, por falta de aferição ou até mesmo pela ausência de tal sinal clínico nesta casuística com grau leve a moderado.

Já com relação aos sintomas nasais, notou-se predomínio de obstrução nasal (62\%), irritação nasal (54\%) e secreção nasal (45\%). Considerando sintomas das vias aéreas superiores, congestão nasal esteve presente em apenas 3,7\% em Lovato A, et al.( 2020), rinorreia foi encontrada por Chen N, et al. (2020) em 4\% dos pacientes e por Lima MA, et al. (2020), em 49,1\%. Concordando com este estudo, os sintomas nasais (obstrução nasal, espirros, coriza, rinorreia purulenta, prurido nasal e/ou ardência nasal) foram relatados por 111 pacientes (43,9\%) e dor de garganta por 16 (6,3\%) por Kosugi EM, et al. (2020).

Estudos iniciais sugeriram vários mecanismos possíveis para o desenvolvimento de anosmia, incluindo síndrome da fenda olfatória, obstrução nasal e rinorreia, tempestade de citocinas, dano direto a receptores olfatórios de neurônios e comprometimento dos centros de percepção olfatória cerebrais (BILINSKA K, BUTOWT R, 2020).

A síndrome da fenda olfatória foi relatada apenas em casos raros. Por outro lado, a rinorreia ou obstrução nasal associada, que poderia bloquear o fluxo aéreo nasal, é conhecida por ser observada com muito menos frequência na COVID-19. Além disso, não há dados sólidos que sustentem o rápido dano às áreas corticais olfatórias do cérebro, considerando que a anosmia é frequentemente detectada muito cedo na doença, tanto em casos leves quanto em pacientes assintomáticos. Finalmente, uma causa mais óbvia de anosmia associada a COVID-19 pode ser a de dano direto aos receptores de neurônios olfatório, já que outros coronavírus humanos foram previamente descritos por se ligarem diretamente a esses receptores (BILINSKA K, BUTOWT R, 2020).

Recentemente, houve maior empenho na identificação da importância das células de sustentação, presentes no epitélio olfatório, na gênese das alterações olfatórias, visto que expressam altos níveis de ECA2 e TMPRSS2. Além das células de sustentação, células progenitoras / tronco e glândulas de Bowman também expressam ECA-2 e é amplamente aceito que a infectividade do SARS-CoV-2 e sua entrada no corpo dependem da ligação a esses receptores. Levando todos os fatos acima em consideração, o comprometimento das células de sustentação causado pela infecção viral pode facilmente inibir a percepção de odorantes em receptores olfatórios adjacentes (BILINSKA K, BUTOWT R, 2020). 
Neste estudo, 84\% (64/76) dos pacientes relatou alterações olfatórias, sendo a maioria (63\%) anosmia. A percepção das alterações no olfato ocorreu em $3,7 \pm 2,1$ dias no grupo que relatou anosmia e em 3,8 $\pm 2,5$ dias no grupo com hiposmia. Dos 64 pacientes que relataram alterações de olfato, $58(90 \%)$ já apresentavam melhora do quadro no momento da pesquisa. O tempo para recuperação foi significativamente maior entre os pacientes que apresentaram anosmia em comparação àqueles que apresentaram hiposmia $(17,8 \times 12,8$, respectivamente, $p<0,05$ ) e apenas 1 paciente realizou tratamento com treinamento olfativo. Sabe-se que a prevalência destes distúrbios olfatórios é expressiva, porém bastante variável, oscilando de acordo com o território de estudo e o tipo de protocolo de pesquisa adotado.

Habitualmente, a prevalência de hiposmia costuma ser mais do que o dobro da anosmia (BRAMERSON A, et al., 2004). Porém, os estudos de Hopkins C, et al. (2020) e Lechien JR, et al. (2020) mostraram que 74,4 e 76,9\%, respectivamente, das perdas do olfato relacionadas à COVID-19 eram de anosmia, e não de hiposmia. Em concordância, para Kosugi EM, et al. (2020), 83,4\% das queixas de olfato eram de anosmia. Assim, estima-se que até $2 / 3$ dos pacientes tenham alterações olfativas, na maioria dos casos, descritas como anosmia súbita e, menos frequentemente, hiposmia (LUERS JC, et al., 2020).

Além do questionário a respeito das alterações de olfato, foi aplicado nos pacientes o teste olfatório ProFono, no qual foi possibilitado testar 9 aromas diferentes (dentre 15 disponíveis) e de fácil identificação. Não houve diferença na pontuação do teste olfativo entre os pacientes com histórico de anosmia, hiposmia ou sem alteração olfativa. Alguns elementos de confusão podem ter interferido nesse resultado, como a falta de atenção dos pacientes; a dessensibilização nasal após teste com múltiplos aromas diferentes, falhas referentes a sensibilidade do teste utilizado ou a melhora do quadro no momento da avaliação.

Ao avaliar terapêutica instituída para cada paciente, $64 \%$ (49/76) realizou algum tipo de tratamento medicamentoso durante o quadro. Diversos medicamentos foram citados, de forma isolada ou combinada. A azitromicina foi o medicamento mais utilizado, presente em $71 \%$; os corticoides orais/tópicos foram prescritos em $41 \%$ e a hidroxicloroquina em $29 \%$. Dentre as combinações terapêuticas possíveis, a associação entre azitromicina e hidroxicloroquina foi a mais comum. Não houve diferença entre o número de sintomas relatados quando se comparou os pacientes que não fizeram uso de nenhuma medicação $(N=27)$ com o restante $(N=$ 49).

Os seguintes tratamentos mais considerados para pacientes com infecção por COVID-19 incluem paracetamol (62,4\%); antiinflamatórios não esteroidais $(9,8 \%)$; irrigações salinas nasais $(9,6 \%)$; cloroquina (7,9\%); mucolíticos (5,0\%); e corticosteroides orais (1,4\%, com antibióticos concomitantes) (KOSUGI EM, et al., 2020). Embora vários tratamentos tenham sido tentados na anosmia pós-viral (por exemplo, corticosteroides, zinco, vitamina A), nenhuma terapia farmacológica foi estabelecida até o momento (HUMMEL T, et al., 2020).

Nesta pesquisa, notou-se que não houve diferença de pontuação no teste olfativo oferecido entre pacientes que realizaram ou não algum tipo de tratamento prescrito, o que sugere que o curso natural das alterações olfatórias tem pouca resposta à terapêutica conhecida e adotada na atualidade, pelo menos nas observações de curto prazo. O uso contínuo de esteroides nasais, por exemplo, não mostrou diferença no surgimento de alteração de olfato, fosse parcial ou total (JOFFILY L, et al., 2020).

O treinamento olfativo, que oferece estímulos odorantes ao sistema olfatório e contribui para a regeneração do mesmo pode ser, alternativamente, empregado com duas sessões diárias nas quais o paciente inspira por 15 segundos quatro odores diferentes, perfazendo uma duração mínima de seis meses de terapia. Entretanto, os resultados desta terapia têm sido, na maioria das vezes, modestos e os pacientes idealmente devem ser acompanhados por um otorrinolaringologista (SOLER ZM, et al., 2020). Apesar de ser uma alternativa de tratamento, esta modalidade foi adotada uma única vez entre os pacientes entrevistados por esta pesquisa, provavelmente por conta da difícil adesão a esta modalidade.

Analisando somente pacientes COVID-19 positivos, Kosugi EM, et al. (2020), observou que a hiposmia apresentou recuperação total com mais facilidade do que a anosmia; que não houve diferença na recuperação total da perda súbita do olfato em função dos diferentes tratamentos usados e que não houve relação entre recuperação total da perda súbita do olfato em função da presença ou ausência de outros sintomas, concordando com as conclusões presentes neste estudo. 


\section{CONCLUSÃO}

A infecção pela COVID-19 modificou drasticamente o andamento global, sob todos os setores da sociedade, e com este contexto pandêmico e até então desconhecido, observou-se uma corrida para busca de informações a respeito da doença, sua epidemiologia e formas de tratamento. Este estudo possibilitou trazer informações regionalizadas sobre o norte do Brasil, no qual se evidencia que na população estudada 0 gênero feminino foi o mais prevalente; com idade média de 42 anos, havendona maioria positividade para os testes da COVID-19. Rinite e sinusite foram as comorbidades mais referidas. Anosmia foi a disfunção olfatória mais comum. Os pacientes foram tratados em sua maioria com azitromicina, sem que isso, no entanto, interferisse na história natural das alterações olfatórias.

\section{AGRADECIMENTOS}

Aos serviços de medicina do trabalho do HUJBB e HUBFS, agradecemos por ceder seus espaços físicos para realização da pesquisa.

\section{REFERÊNCIAS}

1. ANSARIN M. Surgical management of head and neck tumours during the SARS-CoV (Covid-19) pandemic. Acta Otorhinolaryngology Italica, 2020; 40(2): 152-153.

2. BILINSKA K, BUTOWT R. Anosmia in COVID-19: A Bumpy Road to Establishing a Cellular Mechanism. ACS Chemical Neuroscience, 2020; 11(15): 2152-2155.

3. BRAMERSON A, et al. Prevalence of olfactory dysfunction: the skövde population-based study. The Laryngoscope, 2004; 114(4): 733-737.

4. BRASIL. Ministério da Saúde. Secretaria de Atenção Primária à Saúde. Protocolo de manejo clínico do coronavírus na atenção primária à saúde. 2020. Disponível em: https://saude.rs.gov.br/upload/arquivos/202004/14140606-4-ms-protocolomanejo-apsver07abril.pdf. Acessado em: 06 de fevereiro de 2021.

5. CAO QD, et al. The origin, transmission and clinical therapies on coronavirus disease 2019 (COVID-19) outbreak - an update on the status. Military Medical Research, 2020; 7(1): 1-11.

6. CHEN N, et al. Epidemiological and clinical characteristics of 99 cases of 2019 novel coronavirus pneumonia in Wuhan, China: a descriptive study. The Lancet, 2020; 395: 507-13.

7. DUARTE MMS, et al. Descrição dos casos hospitalizados pela COVID-19 em profissionais de saúde nas primeiras nove semanas da pandemia, Brasil, 2020. Epidemiologia e Serviços de Saúde, 2020; 29(5): e2020277.

8. GUAN WJ, et al. Clinical characteristics of coronavirus disease 2019 in China. The New England Journal of Medicine, 2020; 382 : 1708-1720.

9. HOPKINS C, et al. Presentation of new onset anosmia during the Covid-19 pandemic. Rhinology International Journal, 2020; 58(3): 295-298.

10. HUMMEL T, et al. Olfactory dysfunction in COVID-19: diagnosis and management.Journal of the American Medical Association, 2020; 323(24): 2512-2514.

11. JOFFILY L, et al. The close relationship between sudden loss of smell and Covid-19. Brazilian Journal of Otorhinolaryngology, 2020; 86(5): 632-638.

12. KOSUGI EM, et al. Incomplete and late recovery of sudden olfactory dysfunction in COVID-19. Brazilian Journal of Otorhinolaryngology, 2020; 86: 490-496.

13. LECHIEN JR, et al. Olfactory and gustatory dysfunctions as a clinical presentation of mild-to-moderate forms of the coronavirus disease (COVID-19): a multicenter European study. European Archives of Oto-Rhino-Laryngology, 2020; 277(8): 2251-2261.

14. LIMA MA, et al. Smell dysfunction in COVID-19 patients: More than a yes-no question. Journal of the Neurological Sciences, 2020; 418: 117107.

15. LOVATO A, et al. Clinical Presentation of COVID-19: A Systematic Review Focusing on Upper Airway Symptoms. Ear, Nose \& Throat Journal, 2020; 99(9): 1-8.

16. LUERS JC, et al. Die COVID-19-Pandemie und das HNO-Fachgebiet: Worauf kommt es aktuell an? Laryngo-Rhino-Otologie, 2020; 99(5): 287-291.

17. MENNI C, et al. Loss of Smell and Taste in Combination with Other Symptoms Is a Strong Predictor of COVID-19 Infection. Epidemiology, 2020; 7: 1-23.

18. MOEIN ST, et al. Smell dysfunction: a biomarker for COVID-19. International Forum of Allergy and Rhinology, $2020 ; 10(8)$ : $944-950$.

19. MUSTAFA MWM. Audiological profile of asymptomatic Covid-19 PCR-positive cases. American Journal Otolaryngology, 2020; 41 (3): $1-3$.

20. NETLAND J, et al. Severe acute respiratory syndrome coronavirus infection causes neuronal death in the absence of encephalitis in mice transgenic for human ACE2. Journal of Virology, 2008; 82(15): 7264-7275.

21. PADERNO A, et al. Smell and taste alterations in COVID-19: a cross-sectional analysis of different cohorts. Internartional Forum of Allergy and Rhinology, 2020; 10(8): 955-962.

22. SOLER ZM, et al. A primer on viral-associated olfactory loss in the era of COVID-19. International Forum of Allergy \& Rhinology, 2020; 10(7): 814-820.

23. TORRETTA S, et al. Why Italian ENT physicians should be aware of SARS-CoV-2. Acta Otorhinolaryngolica Italica, 2020; 40(2): 152-153.

24. VILLALBA NL, et al. Anosmia and dysgeusia in the absence of other respiratory diseases: should COVID-19 infection be considered? European Journal of Case Report in Internal Medicine, 2020; 7(4): 1641.

25. WANG D, et al. Clinical Characteristics of 138 Hospitalized Patients With 2019 Novel Coronavirus-Infected Pneumonia in Wuhan, China. Journal of the American Medical Association, 2020; 323(11): 1061-1069.

26. XIE J, et al. Critical care crisis and some recommendations during the COVID-19 epidemic in China. Intensive Care Medicine, 2020; 46(5): 837-840.

27. XYDAKIS MS, et al. Smell and taste dysfunction in patients with COVID-19. The Lancet Infectious Diseases, 2020; 20 (9): 1015-1016.

28. YAN CH, et al. Association of chemosensory dysfunction and Covid-19 in patients presenting with influenza-like symptoms. International Forum of Allergy \& Rhinology, 2020; 10(7): 806-813.

29. ZHANG JJ, et al. Clinical characteristics of 140 patients infected with SARS-CoV-2 in Wuhan, China. Allergy, 2020; 75(7): 17301741. 